Rev. Elev. Méd. vét. Pays trop., 1974, 27 (3) : 363-369

\title{
Étude de la germination de quelques graminées de savane et de ses rapports avec leur répartition
}

\author{
par P. GRANIER (*) et Y. CABANIS \\ (avec la collaboration technique de R. RAZAFINDRATSITA)
}

\begin{abstract}
RESUME
Les auteurs ont étudié les capacités de germinations des principales graminées de savane. Une expérimentation sur l'influence des facteurs écologiques montre que ces espèces ont une capacité de germination élevée, ce qui explique leur grande dispersion. La comparaison des vitesses de germinations permet de préciser le rôle des arêtes et la répartition de ces espèces dans des milieux différents.

$\mathrm{La}$ régénération des pâturages ne dépend donc pas de la capacité de germination, mais comme le montre un contrôle de l'évolution des plantules en savane, de l'kouverture " du milieu dans laquelle le feu et l'action du bétail jouent un rôle essentiel.
\end{abstract}

\section{INTRODUCTION}

Parmi les facteurs du déterminisme đes écosystèmes, les facteurs biotiques semblent jouer un rôle essentiel. Dans un milieu dont les caractéristiques physiques sont homogènes (sol, climat) la dynamique des associations végétales est soumise à l'influence de facteurs biologiques qui, en modifiant les conditions de la compétition interspécifique, permettent à certaines espèces de devenir dominantes ou envahissantes.

Une association végétale en savane voit son équilibre constamment remis en cause, alors qu'on a pensé pendant longtemps que ce milieu était statique, la flore étant pauvre, souvent constituée d'espèces banales pantropicales offrant peu d'intérêt pour une étude phytosociologique.

I.E.M.V.T., Laboratoire Central de l'Elevage, B.P. 862, Tananarive, République Malgache.

(*) Adresse actuelle: Laboratoire de l'Elevage, B.P. 485, Niamey, République du Niger.
En fait, la strate graminéenne en savane est soumise chaque année à des variations importantes qui intéressent la biomasse, le recouvrement et l'évolution de la matière organique.

Les principaux facteurs susceptibles de modifier l'équilibre de la végétation sont, très sommairement :

- L'influence du pâturage sélectif;

- L'influence des feux courants;

- Le mode d'exploitation (charge, rythme, modifications des caractéristiques du sol);

- L'influence de l'homme qui « en ouvrant » la strate herbacée élimine ou au contraire favorise certains ligueux.

\section{LA BIOLOGIE DES ESPECES}

Des différences importantes existent au niveau de la longévité (annuelles, bisannuelles, vivaces à des degrés différents), du mode d'occupation de l'espace aérien et souterrain 
(géophytes, hémicryptophytes, chaméphytes) et des besoins spécifiques en eau, minéraux et matière organique.

La compétition entre les espèces est donc sans cesse modifiée et la conquête du milieu met en jeu des facteurs parmi lesquels la capacité de reproduction, de dissémination des diaspores et leur faculté de germination sont essentiels. L'analyse des relations entre la germination des graminées et leur répartition dans la formation nécessite l'étude, d'une part de l'action sélective du milieu et d'autre part des facultés germinatives dans différentes conditions des principales espèces.

Une étude expérimentale des conditions de la germination de six espèces fondamentales du pâturage naturel a été effectuée. Les buts en étaient :

- L'étude de la capacité de germination;

- La recherche et mise en évidence des dormances embryonnaires et des inhibitions tégumentaires et appendicielles (arêtes);

- L'étude de l'influence des différents facteurs écologiques (prétraitements).

\section{PROTOCOLE EXPERIMENTAL}

Les graines récoltées au cours de saison sèche ont été soumises:

— soit à un prétraitement pendant la période qui a précédé la germination,

- soit à un traitement au moment même où on les mettait à germer.

On s'est efforcé de reconstituer en laboratoire les effets des principaux facteurs écologiques auxquels les semences sont soumises dans la nature.

Ces facteurs sont: l'ensoleillement, l'humidité, la chaleur, le fcu, la microfaune et microflore du sol qui détruisent les téguments.

\section{a) Les prétraitements}

L'action du soleil: Les semences ont été exposées au soleil à même le sol pendant trois mois.

L'action de l'humidité: A été obtenue en maintenant les graines dans une atmosphère saturée d'humidité soư une bâche en matière plastique pendant 8 jours.
L'action de la chaleur :

- Chaleur des feux courants. Les graines ont été placées dans une étuve, et soumises à une température de $200^{\circ}$ pendant 3 minutes, temps pendant lequel rayonne la chaleur en savane par vent moyen.

- Elévation de température du sol nu due à l'énergie solaire. Les semences ont été soumises à une température de $60^{\circ}$ pendant une longue période de 8 jours, ce qui correspond environ à la somme des températures que reçoit une graine en savane.

\section{b) Les traitements}

L'action du feu : Les feux courants de saison sèche pouvant intervenir à 2 époques distinctes, soit précoce, soit tardive, ils concernent des semences qui n'ont pas eu le temps d'être enterrées ou au contraire des semences dont seule l'arête est visible. C'est pour cette raison qu'on a utilisé le feu, soit sur des graines déposées sur la surface des cristallisoirs, soit enterrées et dans ce cas le feu n'a intéressé que l'arête.

La dénudation : Les téguments ont été enlevés sous la loupe binoculaire ou selon les espèces en les grattant au papier de verre.

\section{c) La germination}

Les lots de 100 graines ont été placés dans un germoir où la température était maintenue à $24^{\circ}$ et l'atmosphère saturée en humidité.

Une répétition a été effectuée à 1 mois d'intervalle. Les résultats sont les moyennes des deux expérimentations.

\section{d) Observations}

Afin de caractériser le taux de germination, mais également la rapidité de germination en fonction du temps (coefficient de vélocité) des contrôles ont été faits à 6,12 et 16 jours.

\section{RESULTATS}

\section{A. Expérimentation au laboratoire (tableau $\mathrm{n}^{\circ} \mathrm{I}$ )}

Il apparaît, d'une manière générale, que le pouvoir germinatif de ces six graminées dominantes des pâturages de savane est excellent 
TABLEAU N ${ }^{\circ}$ I. - Etude des capacités de germination (en p.100)

\begin{tabular}{|c|c|c|c|c|c|c|c|c|c|c|c|c|c|c|c|c|c|c|}
\hline & \multicolumn{3}{|c|}{$\begin{array}{l}\text { Aristida } \\
\text { mufescens }\end{array}$} & \multicolumn{3}{|c|}{$\begin{array}{l}\text { Chrysopogor } \\
\text { serruzatis }\end{array}$} & \multicolumn{3}{|c|}{$\begin{array}{l}\text { Heteropogon } \\
\text { contortus }\end{array}$} & \multicolumn{3}{|c|}{$\begin{array}{c}\text { Fyparphenia } \\
\text { rufa } \\
\end{array}$} & \multicolumn{3}{|c|}{$\begin{array}{l}\text { Imperata } \\
\text { cytindrica }\end{array}$} & \multicolumn{3}{|c|}{$\begin{array}{l}\text { Byperthelia } \\
\text { dissoluta }\end{array}$} \\
\hline \multirow[t]{2}{*}{ Traitement } & \multicolumn{3}{|c|}{$\begin{array}{l}\text { Pourcentage } \\
\text { de levées }\end{array}$} & \multicolumn{3}{|c|}{$\begin{array}{c}\text { Pourcentage } \\
\text { de levées }\end{array}$} & \multicolumn{3}{|c|}{$\begin{array}{l}\text { Pourcentage } \\
\text { de levêes }\end{array}$} & \multicolumn{3}{|c|}{$\begin{array}{l}\text { Pourcentage } \\
\text { de levëes }\end{array}$} & \multicolumn{3}{|c|}{$\begin{array}{l}\text { Pourcentage } \\
\text { de levées }\end{array}$} & \multicolumn{3}{|c|}{$\begin{array}{c}\text { Pourcentage } \\
\text { de levées }\end{array}$} \\
\hline & $6 \mathrm{j}$ & $12 j$ & $16 j$ & $6 j$ & $12 \mathrm{j}$ & $16 j$ & $6 j$ & $12 \mathrm{j}$ & $16 \mathrm{j}$ & $6 j$ & $12 j$ & $16 j$ & $6 j$ & $12 \mathrm{j}$ & $16 \mathrm{j}$ & $6 j$ & $12 \mathrm{j}$ & $16 \mathrm{j}$ \\
\hline $\begin{array}{l}\text { Têmoin } \\
\text { sans traitement }\end{array}$ & 0 & 16 & 36 & 0 & 27 & 30 & 14 & 31 & 40 & 25 & 35 & 40 & 1 & 47 & 75 & 10 & 12 & 17 \\
\hline $\begin{array}{l}\text { Feu/graines } \\
\text { en surface }\end{array}$ & 0 & 1 & 3 & 20 & 32 & 43 & 18 & 23 & 23 & 15 & 19 & 19 & 9 & 17 & 18 & 12 & 17 & 22 \\
\hline $\begin{array}{l}\text { Feu/graines } \\
\text { enterrëes } \\
\text { (sauf les arêtes) }\end{array}$ & 0 & 4 & 6 & 9 & 13 & 15 & 60 & 64 & 64 & 6 & 10 & 15 & 2 & 13 & 13 & 40 & 42 & 44 \\
\hline $\begin{array}{l}\text { Chaleur } 200^{\circ} \\
\text { pendant } 3 \mathrm{mn}\end{array}$ & 0 & 0 & 0 & 0 & 0 & 0 & 0 & a & 0 & 0 & 0 & 0 & 0 & 0 & 0 & 0 & 0 & 0 \\
\hline $\begin{array}{l}\text { Chaleur } 60^{\circ} \\
\text { pendant } 8 \text { jours }\end{array}$ & 1 & 14 & 22 & 57 & 73 & 80 & 18 & 26 & 29 & 2 & 2 & 3 & 27 & 52 & 55 & 15 & 17 & 22 \\
\hline $\begin{array}{l}\text { Exposition au } \\
\text { soleil pendant } \\
3 \text { mois }\end{array}$ & 6 & 39 & 52 & non & rêal & isë & 35 & 46 & 49 & 17 & 20 & 22 & 33 & 45 & 47 & 30 & 32 & 37 \\
\hline $\begin{array}{l}\text { Atmosphère saturée } \\
\text { pendant } 8 \text { jours }\end{array}$ & 23 & 25 & 25 & 20 & 28 & 48 & 20 & 30 & 37 & 20 & 21 & 21 & 44 & 56 & 58 & 15 & 25 & 27 \\
\hline $\begin{array}{l}\text { En1èvement des } \\
\text { arêtes }\end{array}$ & 0 & 13 & 19 & 24 & 37 & 48 & 57 & 59 & 84 & 22 & 22 & 25 & non & rēal & isê & 41 & 52 & 74 \\
\hline Dënudation totale & 7 & 18 & 19 & 27 & 40 & 44 & 53 & 75 & 83 & 31 & 32 & 32 & 51 & 92 & 92 & 42 & 44 & 47 \\
\hline
\end{tabular}

puisque, sauf pour Hyparrhenia (40 p. 100) il se situe au-dessus de 50 p. 100 , ce qui est très élevé pour des graines qui n'ont pas été sélectionnées. Ces espèces ont donc, si les conditions sont favorables, un fort pouvoir de dispersion et de réensemencement, ce sont des espèces à aire étendue.

L'énergie de germination (*) est moyenne. Il faut entre 6 et 12 jours de latence pour la plupart des objets pour obtenir 50 p. 100 de levées. La germination est plus lente pour les témoins (sans traitement). Par contre l'énergie la plus faible ( $<6$ jours) se rencontre dans le cas des graines dénudées qui atteignent rapidement des taux élevés. Les semences sont donc protégées par leurs enveloppes (sauf Aristida) et la germination ne se déclenche que lorsque l'eau a atteint l'embryon.

La capacité de germination varie avec les traitements et si un facteur est négatif pour toutes les espèces (température de $200^{\circ}$ ) chacune d'entre elles est favorisée par un traitement en particulier :

(*) Exprimée par le temps nécessaire pour atteindre 50 p. 100 de la capacité de germination. Lang (1965), Côme (1970).
Aristida : ensoleillement

Chrysopogon: chaleur

Heteropogon : enlèvement des arêtes

Hyperthelia: enlèvement des arêtes

Imperata : dénudation totale

Heteropogon: dénudation totale.

Par contre Hyparrhenia n'a donné aucune réponse aux traitements.

\section{Comportement des différentes espèces}

\section{Discussion}

\section{- Aristida rufescens}

Pouvoir germinatif : moyen

Energie germinative : de l'ordre de 10 jours.

Capacité germinative: on remarque l'influence favorable de l'ensoleillement et de l'humidité, et l'absence de réponse à l'ablation des téguments, soit par le feu ou mécaniquement. La protection tégumentaire est donc faible et l'arête ne joue aucun rôle inhibiteur, mais permet la dispersion et la fixation de la graine sur les sols squelettiques. L'action du 
soleil semble indispensable à la maturation et cela est à rapprocher de la persistance de la diaspore sur les chaumes pendant toute la saison sèche. C'est une espèce du domaine forestier, originaire des stations rocheuses, ensoleillées, soumises à des dessiccations brusques. Elle ne présente pas les caractéristiques des espèces savanicoles.

\section{- Chrysopogon serrulatus}

Pouvoir germinatif : moyen à fort

Energie germinative: de l'ordre de 6 jours.

Capacité germinative. L'influence favorable de la chaleur, de l'humidité et de l'absence de téguments semble confirmer les résultats d'une expérimentation antérieure qui nous avait amené à supposer l'existence d'une inhibition d'ordre chimique, les graines du Chrysopogon ne germant qu'après un séjour prolongé dans le sol.

L'aire de cette espèce est située sur les pentes où les sols décapés subissent des variations brusques d'humidité : elle se comporte comme une espèce de zone sèche et ne germe que lorsque la saison des pluies est bien installée.

\section{- Heteropogon contortus}

Pouvoir germinatif : moyen à bon

Energie germinative: de l'ordre de 6 jours.

Capacité germinative. L'influence de l'arête est déterminante. Pendant la saison sèche elle joue un rôle dans la dispersion de la diaspore en s'accrochant sur de nombreux supports, puis lorsque la graine tombe sur le sol, elle permet, une fois le callus calé sur le support, à celle-ci de pénétrer dans le sol. Les variations de l'humidité de l'air mobilisent la colonne qui imprime à la graine un mouvement de rotation. Il semble que pendant cette période, l'arête inhibe la germination en empêchant la pénétration de l'eau jusqu'à l'embryon. Ensuite, lorsque la graine s'est enfoncée, l'arête est détruite par le feu, ou arrachée par le piétinement du bétail, et l'inhibition peut s'effectuer. Cette espèce réagit bien à tous les traitements, elle a une très grande dispersion due à son bon pouvoir germinatif et à ses exigences modestes sur le plan de la fertilité des sols.

Elle a tendance à remplacer Hyparrhenia rufa dans les savanes brûlées trop fréquemment, étant beaucoup mieux adaptée au feu que cette dernière.

\section{- Hyparrhenia rufa}

Pouvoir germinatif : moyen à médiocre

Energie germinative: de l'ordre de 6 jours, donc faible.

Capacité germinative. Bien que munie d'une arête comme Heteropogon, cette espèce n'a pas le même comportement. Elle paraît indifférente aux divers traitements. Elle germe en surface, mais le développement de la plantule exige que le sol soit ameubli, bicn pourvu en matière organique. Elle ne résiste pas au dessèchement superficiel. C'est une espèce des sols riches, des jachères. Ses exigences se manifestent au niveau de la plantule, ce qui explique qu'elle soit remplacée par Heteropogon dès que l'horizon superficiel s'appauvrit sous l'influence du feu, du lessivage ou du surpâturage.

\section{- Imperata cylindrica}

Pouvoir germinatif : très élevé

Energie germinative : de l'ordre de 16 jours, donc très élevée.

Capacité de germination. On note l'influence favorable de la dénudation et de la mise au contact de l'humidité, et l'action défavorable du feu. Cette espèce a un très grand pouvoir germinatif en milieu humide. La graine est protégée par son enveloppe cotonneuse qui, une fois imbibée, permet une germination rapide en maintenant une atmosphère humide autour de l'embryon. C'est une espèce pionnière qui apparâtt après le défrichement de la forêt, lorsque le sol, riche en matière organique, tamponne les variations des réserves en eau. Sa dispersion est limitée parce que la fructification est accidentelle, déclenchée par un déséquilibre du milieu (feu) et que la plantule ne se développe bien qu'en jachère, ou si la savane est en défens (mulch).

\section{- Hyperthelia dissoluta}

Pouvoir germinatif : bon à élevé

Energie germinative: inférieure à 6 jours.

Capacité de germination. Le comportement de cette espèce est très voisin de celui d'Heteropogon. Sur le plan de la phytosociologie, elle se substitue à Hyparrhenia rufa, lorsque la teneur du sol en matière organique diminue. 


\section{B. Relevés de germinations dans Ie milieu}

\section{- Influence de l'exploitation}

Une étude des germinations a été effectuée en savane afin de préciser l'influence des facteurs écologiques biotiques (le feu et le pâturage) sur l'évolution des plantules.

\section{a) Influence $d u$ feu (tableau $\mathrm{n}^{\circ}$ II)}

Les relevés du tableau $n^{\circ}$ II montrent que le passage du feu en savane favorise la germination des graminées. La mise en défens provoque la formation d'un mulch (*) qui modifie les conditions d'éclairement et favorise la germination des espèces appartenant à d'autres familles. Il y a une augmentation de la productivité primaire mais une diminution des potentialités en matière d'élevage.

Les graminées savanicoles ont besoin pour se maintenir que le milieu soit régulièrement « ouvert» par le passage du feu qui réalise une minéralisation brutale de la matière organique et met à la disposition des racines les éléments qui favorisent le démarrage rapide des nouveaux cycles et l'occupation de l'espace.

Le feu à contre-saison permet le développement des plantules grâce aux réserves en eau du sol.

Heteropogon est mieux adapté au feu que Hyparrhenia, ce qui explique sa plus grande dispersion.

b) Influence du mode d'exploitation (tableau $\mathrm{n}^{\circ}$ UII)

Le contrôle des germinations dans une savane à Hyparrhenia et Heteropogon soumise à des traitements différents montre que les germinations sont abondantes dès que le recouvrement diminue (ouverture du milieu). La mise en défens ou la sous-exploitation ralentissent le développement des savanicoles hémicryptophytes.

TABLEAU N$N^{\circ} I I .-$ Influence du feu sur les germinations (Relevé fait en fin de saison des pluies-1972)

\begin{tabular}{|c|c|c|c|c|c|c|c|c|c|c|}
\hline Objet & $\begin{array}{l}\text { Relevé } \\
\text { numëro }\end{array}$ & Heteropogon & Hyparrhenia & Amiatida & Diverseg & $\begin{array}{c}\text { Total } \\
\text { graminées }\end{array}$ & Légumineuse & Cypéracées & Diverses & $\begin{array}{l}\text { Tota1 } \\
\text { globa1 }\end{array}$ \\
\hline \multirow{3}{*}{$\begin{array}{l}\text { En } \\
\text { Dếfens }\end{array}$} & 1 & & & & & 0 & 157 & 4 & 2 & 163 \\
\hline & 16 & 2 & & & & 2 & 2 & 4 & 1 & 9 \\
\hline & $\theta$ & 4 & & & & 4 & 7 & 15 & 2 & 28 \\
\hline \multirow{3}{*}{$\begin{array}{l}\text { Brûlé } \\
\text { en } \\
\text { saíson } \\
\text { sèche }\end{array}$} & 2 & 268 & 37 & 4 & & 309 & 20 & 7 & 1 & 397 \\
\hline & 12 & 113 & 21 & 2 & & 136 & 1 & 3 & & 140 \\
\hline & 13 & 85 & 13 & 7 & 8 & 113 & 4 & 3 & & 120 \\
\hline \multirow{2}{*}{$\begin{array}{l}\text { Brûle à } \\
\text { contre } \\
\text { saison }\end{array}$} & 3 & 118 & 5 & 2 & 3 & 128 & 4 & 1 & & 133 \\
\hline & 4 & 60 & 10 & 5 & 2 & 77 & 2 & 1 & & 80 \\
\hline
\end{tabular}

en nonbre de germinations par $\mathrm{m}^{2}$.

TABLEAU $N^{0}$ III.-Influence du mode d'exploitation sur les germinations (plantules à 2 talles) (Relevé Eait en fin de saison des pluies 1972).

\begin{tabular}{|c|c|c|c|}
\hline ob jet & 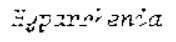 & Heteropogon & Recouvrement \\
\hline $\begin{array}{l}\text { Brûlé en saison } \\
\text { sẽche, pâturé }\end{array}$ & 38 & 210 & Mêdiocre \\
\hline $\begin{array}{l}\text { Brûlé à contre } \\
\text { saison, non pãture }\end{array}$ & 17 & 102 & Mëdiocre \\
\hline Fauché non pâture & 14 & 144 & Bon \\
\hline $\begin{array}{l}\text { Non brûlé } \\
\text { pâturế }\end{array}$ & 20 & 58 & Bon \\
\hline $\begin{array}{l}\text { Non brûlé } \\
\text { surpâturế }\end{array}$ & 13 & 14 & Excellent \\
\hline En dëfens & 4 & 14 & Excellent \\
\hline
\end{tabular}

en nombre de germinat uns par $\bar{m}^{2}$ 
Le surpâturage et l'étalement des touffes au niveau du sol gênent le développement des jeunes talles.

La régénération des graminées ne peut se faire que s'il y a ouverture de l'association, le feu et la pression du pâturage étant indispensables pour maintenir la structure de la formation en équilibre. Le piétinement est utile pour fixer les graines au sol et les maintenir dans des conditions favorables à la germination.

\section{CONCLUSION}

La capacité de dissémination des graminées de savane qui font l'objet de la présente étude est très élevée. Ces espèces produisent une grande quantité de semences dont le pouvoir germinatif est excellent. Leur capacité de germination est adaptée aux facteurs écologiques, ce qui explique que ces espèces ont une aire de dispersion très étendue et que certaines soient pantropicales.

Il n'y a pas d'inhibition qui ne soit levée au cours de la saison sèche qui suit leur formation puisque toutes les semences que nous avons utilisées ont germé l'année de leur récolte. La maturation des graines par exposition au soleil et à la chaleur prolongée améliore le pourcentage de levées.

La protection tégumentaire est d'autant plus forte que l'espèce possède une grande extension. La pénétration de l'eau déclenche immédiatement la germination, mais la vitesse de pénétration de l'eau est plus ou moins rapide selon les espèces et ce caractère permet de distinguer parmi ces graminées que l'on rencontre dans une même formation herbacée, des adaptations plus prononcées au milieu humide. La germination d'Aristida et Imperata est plus liée aux mouvements de l'eau que celle des autres espèces, ce qui autorise à leur attribuer une origine forestière. Des mécanismes protecteurs permettent d'assurer le réensemencement des espèces des milieux soumis à des dessèchements des horizons superficiels comme c'est fréquent en savane. En particulier, les arêtes facilitent la dispersion, la pénétration dans le sol et bloquent la germination jusqu'à l'installation régulière des pluies.

La régénération des pâturages ne dépend donc pas des capacités de germination des espèces fourragères mais essentiellement de l'« ouverture » du milieu. Le contrôle des levées en savane montre que le nombre de plantules est lié à la pression des facteurs biotiques dont les principaux sont le feu et le pâturage. Pour que la structure de l'association végétale se maintienne en équilibre en savane, il est nécessaire que celle-ci soit parcourue régulièrement par les feux et exploitée par le bétail.

Le feu agit par la minéralisation de la matière organique et la destruction de l'écran constitué par les litières. Il favorise la germination des espèces à grande dispersion en détruisant les enveloppes et appendices qui freinent la pénétration de l'eau. Le bétail fixe les graines au sol et facilite leur germination.

La mise en défens prolongée d'une savane aboutit à une modification de sa composition floristique qui se traduit par une diminution de ses potentialités sur le plan fourrager.

\section{SUMMARY}

\section{Study on the germination of some savanna grasses and its relation} with their distribution

The authors studied the germination capacity of the main savanna grasses. Experiments on the effect of the ecological factors show that these species have a high germination capacity, which explains their wide distribution. The part played by the awns and the distribution of the species in various environments can be determined more precisely by comparing the germination rates.

Therefore grassland regeneration does not depend on the germination capacity, but, as can be seen through the control of seedling evolution in the savanna, on the "response" of the environment to the ecological factors in which burning and livestock play an essential part. 


\section{RESUMEN}

\section{Estudio de la germinación de algunas gramíneas de sabana} y de sus relaciones con su repartición

Los autores estudiaron las capacidades de germinaciones de las principales gramíneas de sabana. Una experimentación sobre la influencia de los factores écologicos muestra que estas especies tienen una capacidad de germinación elevada, lo que explica su dispersión importante. La comparación de las velocidades de germinaciones permite precisar el papel de las raspas y la repartición de estas especies en medios diferentes.

Asi la regeneración de los pastos no depiende de la capacidad de germinación sino, como lo muestra un control de la evolución de los brotes en sabana, de la abertura del medio a los factores ecologicos en la cual el fuego y la acción del ganado desempeñan un papel esencial.

\section{BIBLIOGRAPHIE}

1. CÔME (D.). Les obstacles à la germination. Monographies de physiologie végétale. Paris, Masson et Cie, 1970 , p. 162.

2. DELOUCHE (J.C.). Dormancy in seeds of Agropyron smithii, Digitaria sanguinalis and Poa pratensis. Iowa $S t$. coll. J. Sci, 1956, 30 : 348-349.

3. FUJII (T), YOKOHAMA (Y.). Physiology of light-requiring germination in Eragrostis seeds. Pl. cell. Physiol., 1965, 6: 135-144.

4. HESLOP-HARRISON (J.). Photoperiodic effects of sexuality, breeding system and seed germinability in Rottboellia exaltata. Proc. IXth internat. Bot. Cong., Montréal, 1959, 11: 162-163.

5. KILLIAN'(C.), LEMEE (G.), Les xérophytes : leur économie d'eau. Handb., Pflanzenphysıol, $1956,3: 787-824$.

6. LEBRUN (J.). La végétation de la plaine alluviale au Sud du Lac Edouard. Bruxelles, Institut des parcs nationaux Congo Belge, 1947, 800 p.
7. Mc WILLIAM (J. R.), SHANKER (K.), KNOX (R. B.). Effects of temperature and photoperiod on growth and reproductive development in $\mathrm{Hy}$ parrhenia hirta. Aust. J. Agric. Res., 1970, 21 : 557-569.

8. MALCOSTE (R.). Un problème écologique: la germination des semences dans les conditions naturelles. Ann. Biol., 1968, 7 (5-6): 241-274.

9. MARTIN (M.). Influence de la température sur la germination des graines de Phacelia tanacetifolia Benth. C.R. Acad. Sci., 1964, 258, sér., 11 : $1877-1880$

10. MORAT (P.). Contribution à l'étude des savanes du Sud-Ouest de Madagascar. Thèse Doctorat es Sciences Naturelles. Faculté Paris-Sud Orsay, juin 1972, n० 985, série A.

11. ROLLIN (P.). La physiologie de la germination Paris, C.D.U. ed., 1966, 64 p. 